\title{
Relationship between muscle strength and anthropometric, body composition parameters in Japanese adolescents
}

\author{
Nobuyuki Miyatake $^{1^{*}}$, Motohiko Miyachi ${ }^{2}$, Izumi Tabata $^{3}$, Noriko Sakano ${ }^{1}$, Tomohiro Hirao ${ }^{4}$, \\ Takeyuki Numata ${ }^{5}$ \\ ${ }^{1}$ Department of Hygiene, Faculty of Medicine, Kagawa University, Kagawa, Japan; \\ *Corresponding Author: miyarin@med.kagawa-u.ac.jp \\ ${ }^{2}$ National Institute of Health and Nutrition, Tokyo, Japan \\ ${ }^{3}$ Sport and Health Science, Ritsumeikan University, Shiga, Japan \\ ${ }^{4}$ Department of Public Health, Faculty of Medicine, Kagawa University, Kagawa, Japan \\ ${ }^{5}$ Okayama Southern Institute of Health, Okayama, Japan
}

Received 8 November 2011; revised 29 November 2011; accepted 8 December 2011

\section{ABSTRACT}

We investigated the link between muscle strength and anthropometric, body composition parameters in Japanese adolescents. Forty eight men and 189 women, aged 15 - 19 years, were enrolled in this cross-sectional investigation study. Anthropometric and body composition parameters i.e. height, body weight, abdominal circumference, hip circumference and body fat percentage were measured. Muscle strength i.e. grip strength and leg strength were also evaluated. In men, grip strength was positively correlated with height and lean body mass. Leg strength was significantly correlated with height, body weight, hip circumference and lean body mass ( $r=0.708)$. However, leg strength per body weight was negatively correlated with body mass index (BMI), abdominal circumference, hip circumference, body fat percentage $(r=-0.719)$ and body fat mass. In women, grip strength was positively correlated with height. Leg strength was positively correlated with lean body mass ( $r$ $=0.482$ ). Leg strength per body weight was negatively correlated with body fat percentage ( $r=$ -0.457) and body fat mass. Grip and leg strength was positively correlated with height and lean body mass, especially in men. However, leg strength per body weight was closely linked to body fat percentage in both sexes.

Keywords: Anthropometric Parameters; Body Composition; Grip Strength; Leg Strength; Leg Strength per Body Weight

\section{INTRODUCTION}

It is also well known that low and declining muscle strength is associated with increased mortality, independent of physical activity and muscle mass [1]. It has been well reported that there is significant loss in muscle strength with aging [2,3]. Aging is associated with alterations in body composition; there is an increase in body fat percentage and a concomitant decline in lean body mass [4]. Aging, therefore, results in substantial alterations in body composition, with a marked reduction in skeletal muscle mass. Loss of muscle strength may be an important cause of the age-related loss in bone strength resulting in osteoporosis and can also influence the ability to perform simple tasks such as sitting on a chair or visiting the toilet [5]. We have previously evaluated muscle strength, aged 20 - 79 years, and found that agerelated changes in muscle strength were noted. In addition, lower leg strength per body weight was fundamental feature in subjects with obesity [6]. In this respect, it is important to evaluate muscle strength of adolescents for preventing future decline in muscle strength in adults. However, the relation between muscle strength, especially leg strength and anthropometric, body composition parameters still remains to be investigated.

In this study, we evaluated muscle strength i.e. grip strength and leg strength and its relation to anthropome tric, body composition parameters in Japanese adolescents.

\section{SUBJECTS AND METHODS}

\subsection{Subjects}

We used data for 48 men and 189 women, aged 15 19 years, who met the following criteria: 1) received a 
health check-up including health guidance, 2) received anthropometric and body composition measurements, and muscle strength measurements as part of the annual health check-up, 3) received no medications for diabetes, hypertension, and/or dyslipidemia, and 4) provided written informed consent (Table 1).

Ethical approval for the study was obtained from the Ethical Committee of Okayama Health Foundation.

\subsection{Anthropometric and Body Composition Measurements}

Their anthropometric and body composition were evaluated by using the following respective parameters such as, height, body weight, body mass index (BMI), abdominal circumference, hip circumference and body fat percentage. BMI was calculated by weight/[height $]^{2}\left(\mathrm{~kg} / \mathrm{m}^{2}\right)$. Abdominal circumference was measured at the umbilical level in standing subjects after normal expiration [7] and the hip was measured at the widest circumferences over the trochanter in standing subjects after normal expiration. Body fat percentage was measured by an air displacement plethysmograph called the BOD POD Body Composition System (Life Measurement Instruments, Concord, CA) [8,9]. Body fat mass was calculated as follows: body weight $\times$ body fat percentage $\times 100$. Lean body mass was also calculated as follows: body weight -body fat mass.

\subsection{Muscle Strength}

To assess muscle strength, grip and leg strength were measured [6,10]. Grip strength was measured using THP-10 (SAKAI, Tokyo, Japan), while leg strength was measured by COMBIT CB-1 (MINATO, Osaka, Japan).
Isometric leg strength was measured as follows: the subject sat in a chair, grasping the armrest in order to fix the body position. A dynamometer was then attached to the subject's one ankle joint by a strap. The subject extended his or her leg to 60 degrees as described in previous reports $[6,10,11]$ which have also demonstrated good accuracy for this measurement [11]. All muscle strength measurements were recorded in 2 trials, and the better one was employed for analysis. In addition, to standardize the influence of body weight, we calculated the ratio of leg strength to body weight; a 1.0 in leg strength per body weight has been a standard in past studies [11].

\subsection{Statistical Analysis}

Data are expressed as means \pm standard deviation (SD). Pearson's correlation coefficients were calculated and used to test the significance of the linear relationship among continuous variables; stepwise multiple regression analysis was also used.

\section{RESULTS}

Clinical profiles of enrolled subjects are summarized in Table 1. In addition, we evaluated the relationship between anthropometric, body composition parameters and muscle strength (Table 2). In men, grip strength was positively correlated with height and lean body mass. Leg strength was significantly correlated with height, body weight, hip circumference and lean body mass ( $\mathrm{r}=$ 0.708, $p<0.0001$ ) (Figure 1). However, leg strength per body weight was negatively correlated with BMI, abdominal circumference, hip circumference, body fat percentage ( $\mathrm{r}=-0.719, p<0.0001)$ (Figure 2 ) and body fat mass $(\mathrm{r}=-0.623, p<0.0001)$. In women, grip strength

Table 1. Clinical profiles of enrolled subjects.

\begin{tabular}{|c|c|c|c|c|c|c|}
\hline & \multicolumn{3}{|c|}{ Men } & \multicolumn{3}{|c|}{ Women } \\
\hline & Mean \pm SD & Minimum & Maximum & Mean \pm SD & Minimum & Maximum \\
\hline Number of subjects & 48 & & & 189 & & \\
\hline Age & $17.8 \pm 1.2$ & 15 & 19 & $18.4 \pm 1.0$ & 15 & 19 \\
\hline Height (cm) & $170.3 \pm 6.2$ & 154.6 & 181.6 & $157.8 \pm 5.5$ & 135.1 & 175.5 \\
\hline Body weight (kg) & $67.7 \pm 15.3$ & 42.1 & 118.7 & $54.3 \pm 9.3$ & 35.9 & 96.2 \\
\hline Body mass index $\left(\mathrm{kg} / \mathrm{m}^{2}\right)$ & $23.2 \pm 4.6$ & 17.6 & 39.8 & $21.8 \pm 3.7$ & 16.2 & 37.6 \\
\hline Abdominal circumference $(\mathrm{cm})$ & $76.9 \pm 12.8$ & 58.5 & 122.2 & $67.2 \pm 7.6$ & 54.5 & 106.4 \\
\hline Hip circumference & $93.5 \pm 8.2$ & 77.2 & 116.6 & $91.7 \pm 6.6$ & 77.0 & 125.0 \\
\hline Body fat percentage (\%) & $18.0 \pm 7.8$ & 8.7 & 39.0 & $27.1 \pm 6.0$ & 12.4 & 46.1 \\
\hline Body fat mass (kg) & $13.0 \pm 9.1$ & 4.8 & 46.3 & $15.1 \pm 5.9$ & 5.3 & 41.3 \\
\hline Lean body mass (kg) & $54.7 \pm 8.3$ & 32.8 & 72.4 & $39.2 \pm 4.7$ & 29.4 & 56.0 \\
\hline Right grip strength (kg) & $42.8 \pm 8.3$ & 16.2 & 60.0 & $26.7 \pm 5.2$ & 9.3 & 41.1 \\
\hline Left grip strength (kg) & $40.7 \pm 9.0$ & 14.3 & 61.4 & $24.9 \pm 4.5$ & 8.9 & 37.5 \\
\hline Leg strength (kg) & $69.4 \pm 15.1$ & 26.8 & 91.5 & $46.9 \pm 10.1$ & 15.8 & 82.0 \\
\hline Leg strength per body weight & $1.04 \pm 0.22$ & 0.58 & 1.44 & $0.87 \pm 0.18$ & 0.38 & 1.42 \\
\hline
\end{tabular}


Table 2. Relationship between muscle strength and anthropometric, body composition parameters.

\begin{tabular}{|c|c|c|c|c|c|c|c|c|}
\hline & \multicolumn{2}{|c|}{ Right grip strength (kg) } & \multicolumn{2}{|c|}{ Left grip strength (kg) } & \multicolumn{2}{|c|}{ Leg strength (kg) } & \multicolumn{2}{|c|}{ Legs strength per body weight } \\
\hline & r & $p$ & r & $p$ & $\mathrm{r}$ & $p$ & $\mathrm{r}$ & $p$ \\
\hline & & & & & Men & & & \\
\hline Height (cm) & 0.417 & 0.0032 & 0.481 & 0.0005 & 0.534 & $<0.0001$ & 0.065 & 0.6584 \\
\hline Body weight (kg) & 0.203 & 0.1662 & 0.208 & 0.1554 & 0.459 & 0.0010 & -0.394 & 0.0056 \\
\hline Body mass index $\left(\mathrm{kg} / \mathrm{m}^{2}\right)$ & 0.112 & 0.4476 & 0.090 & 0.5412 & 0.352 & 0.0140 & -0.456 & 0.0011 \\
\hline $\begin{array}{l}\text { Abdominal circumference } \\
(\mathrm{cm})\end{array}$ & 0.057 & 0.6984 & 0.030 & 0.8375 & 0.281 & 0.0534 & -0.510 & 0.0002 \\
\hline Hip circumference (cm) & 0.199 & 0.1750 & 0.157 & 0.2855 & 0.434 & 0.0021 & -0.456 & 0.0042 \\
\hline Body fat percentage (\%) & -0.264 & 0.0693 & -0.324 & 0.0248 & -0.081 & 0.5844 & -0.719 & $<0.0001$ \\
\hline Body fat mass (kg) & -0.094 & 0.5238 & -0.109 & 0.4620 & 0.128 & 0.3876 & -0.623 & $<0.0001$ \\
\hline \multirow[t]{2}{*}{ Lean body mass (kg) } & 0.478 & 0.0006 & 0.503 & 0.0003 & 0.708 & $<0.0001$ & -0.046 & 0.7538 \\
\hline & \multicolumn{8}{|c|}{ Women } \\
\hline Height (cm) & 0.404 & $<0.0001$ & 0.430 & $<0.0001$ & 0.236 & 0.0011 & 0.022 & 0.7673 \\
\hline Body weight (kg) & 0.102 & 0.1638 & 0.130 & 0.0736 & 0.368 & $<0.0001$ & -0.341 & $<0.0001$ \\
\hline Body mass index $\left(\mathrm{kg} / \mathrm{m}^{2}\right)$ & -0.073 & 0.3197 & -0.052 & 0.4769 & 0.279 & $<0.0001$ & -0.360 & $<0.0001$ \\
\hline Abdominal circumference $(\mathrm{cm})$ & -0.097 & 0.1830 & -0.074 & 0.3097 & 0.247 & 0.0006 & -0.370 & $<0.0001$ \\
\hline Hip circumference $(\mathrm{cm})$ & 0.077 & 0.2906 & 0.086 & 0.2406 & 0.319 & $<0.0001$ & -0.347 & $<0.0001$ \\
\hline Body fat percentage (\%) & -0.192 & 0.0083 & -0.174 & 0.0164 & 0.042 & 0.5646 & -0.457 & $<0.0001$ \\
\hline Body fat mass (kg) & -0.077 & 0.2945 & -0.054 & 0.4641 & 0.198 & 0.0064 & -0.431 & $<0.0001$ \\
\hline Lean body mass (kg) & 0.300 & $<0.0001$ & 0.328 & $<0.0001$ & 0.482 & $<0.0001$ & -0.134 & 0.0662 \\
\hline
\end{tabular}
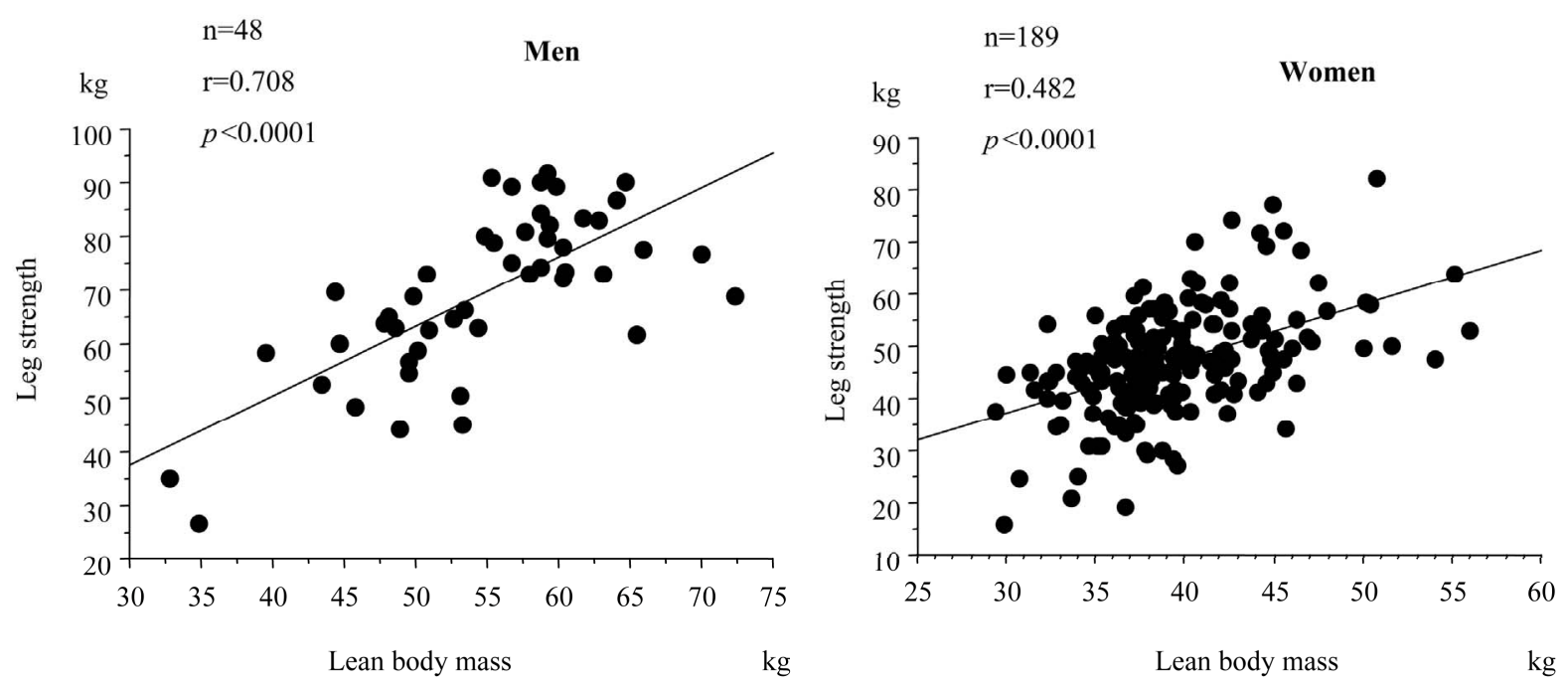

Figure 1. Simple correlation analysis between leg strength and lean body mass. 

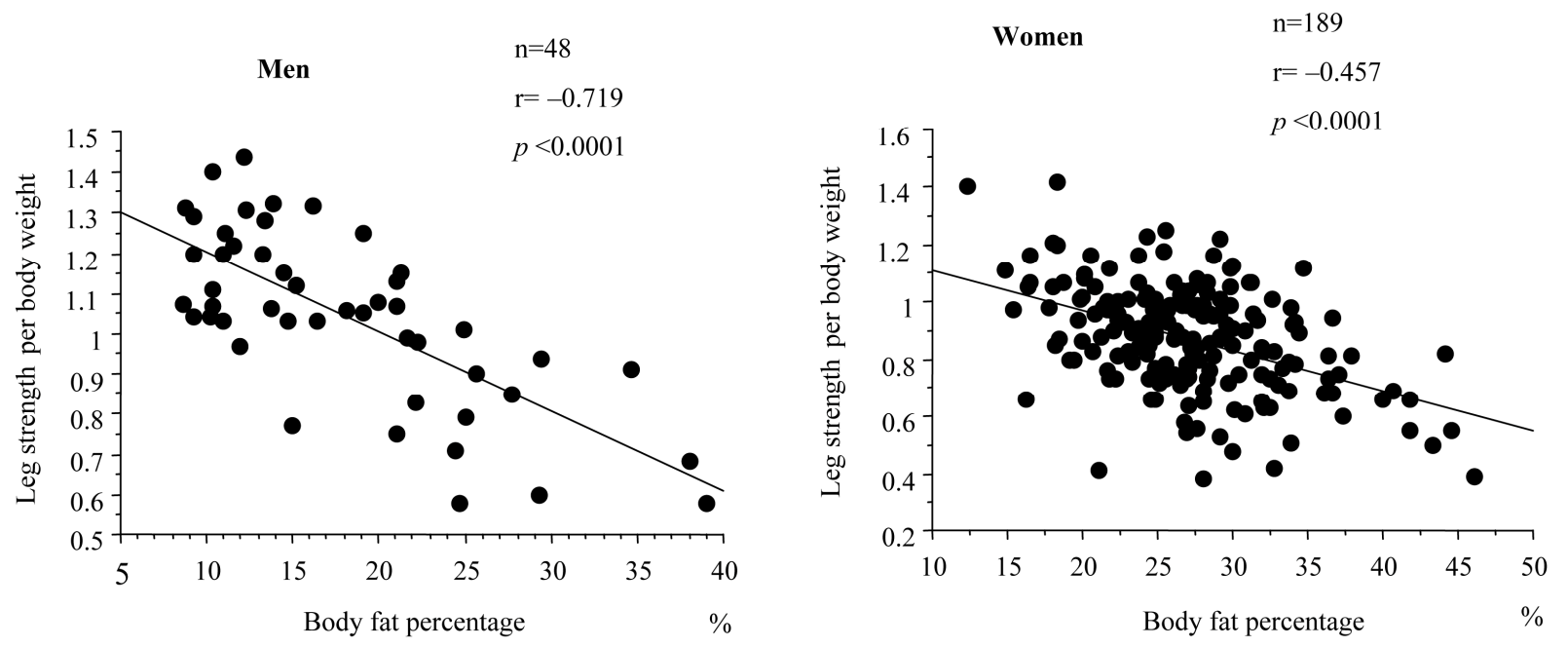

Figure 2. Simple correlation analysis between leg strength per body weight and body fat percentage.

was positively correlated with height. Leg strength was positively correlated lean body mass $(r=0.482, p<$ 0.0001) (Figure 1). Leg strength per body weight was negatively correlated with body fat percentage $(\mathrm{r}=$ $-0.457, p<0.0001$ ) (Figure 2) and body fat mass ( $\mathrm{r}=$ $-0.431, p<0.0001)$.

We also used stepwise multiple regression analysis to evaluate the effect of clinical parameters i.e. age, height, body weight, BMI, abdominal circumference, hip circumference, body fat percentage, body fat mass and lean body mass on leg strength per body weight, and found that age and body fat percentage were significant in men (leg strength per body weight $=0.750+0.036$ (age) 0.020 (body fat percentage), $\mathrm{r}^{2}=0.557, p<0.0001$ ) and only body fat percentage was significant in women (leg strength per body weight $=1.255-0.014$ (body fat percentage), $\left.\mathrm{r}^{2}=0.208, p<0.0001\right)$.

\section{DISCUSSION}

The main finding of this study was that we explored that the relation between muscle strength and anthropometric, body composition parameters, and found that height and lean body mass were closely correlated with grip and leg strength, especially in men. However, leg strength per body weight was negatively correlated with body fat percentage in both sexes.

Jurimae et al. have reported that the most important predictive value for grip strength from the basic anthropometric variables was body height, and skin fold thicknesses was not related to grip strength in prepubertal children aged between 8 and 11 years [12]. They also reported that hand lean mass highly influenced hand grip strength in boys, and moderately but significantly in girls [12]. Koley et al. showed that hand dominance, especially of the right hand, has some close association with the anthropometric variables related to upper extremities in 303 healthy students (height in men: $r=0.275$, height in women: $r=0.200$ ) [13]. Luna-Heredia et al. described that body height is directly correlated with hand grip strength, possibly because this factor is more closely related to the lean body mass [14].

In this study, we also found that height and lean body mass were important factors for grip and leg strength, especially men. However, leg strength per body weight was negatively correlated with body fat percentage. Taken together, it is difficult for Japanese adolescents with lower leg strength per body weight to support the entire body's weight; and also difficult for subjects with lower leg strength per body weight to carry out aerobic exercise i.e. walking and jogging. In addition, we have previously reported that changes in oxygen uptake at VT (per lean body mass) were also correlated with changes in leg strength per lean body mass in adults $[15,16]$. Although aerobic exercise has been advocated as most suitable for reducing fat mass and increasing aerobic exercise level, it is important for subjects with lower leg strength per body weight to maintain or maximize the muscle strength of their lower limbs as well as to carry out aerobic exercise for reducing fat mass and increasing aerobic exercise level in Japanese adolescents.

Potential limitations still remain in this study. First, our study was a cross-sectional and not a longitudinal study. Second, 48 men and 189 women in our study voluntarily underwent measurements: they were therefore more likely to be health-conscious compared with the average person. To show this, further prospective studies are needed to prove the link in the Japanese adolescents.

\section{ACKNOWLEDGEMENTS}

This research was supported in part by Research Grants from the 
Ministry of Health, Labor, and Welfare, Japan. There is no conflict of interest.

\section{REFERENCES}

[1] Metter, E.J., Talbot, L.A., Schrager, M. and Conwit R. (2002) Skeletal muscle strength as a predictor of all-cause mortality in healthy men. The Journals of Gerontology: Series A, 57, B359-B365.

[2] Larsson, L. and Karlsson, J. (1978) Isometric and dynamic endurance as a function of age and skeletal muscle characteristics. Acta Physiologica Scandinavica, 104, 129136. doi:10.1111/j.1748-1716.1978.tb06259.x

[3] Young, A., Stokes, M. and Crowe, M. (1984) Size and strength of the quadriceps muscles of old and young women. European Journal of Clinical Investigation, 14, 282-287. doi:10.1111/j.1365-2362.1984.tb01182.x

[4] Rogers, M.A. and Evans, W.J. (1993) Changes in skeletal muscle with aging: Effects of exercise training. Exercise and Sport Sciences Reviews, 21, 65-102. doi:10.1249/00003677-199301000-00003

[5] Rantanen, T., Guralnik, J.M., Sakari-Rantala, R., Leveille, S., Simonsick, E.M., Ling, S. and Fried, L.P. (1999) Disability, physical activity, and muscle strength in older women: The women's health aging study. Archives of Physical Medicine and Rehabilitation, 80, 130-135. doi:10.1016/S0003-9993(99)90109-0

[6] Miyatake, N., Fujii, M., Nishikawa, H., Wada, J., Shikata, K., Makino, H. and Kimura, I. (2000) Clinical evaluation of muscle strength in 20-79-years-old obese Japanese. Diabetes Research and Clinical Practice, 48, 15-21. doi:10.1016/S0168-8227(99)00132-1

[7] (2005) Definition and the diagnostic standard for metabolic syndrome-Committee to Evaluate Diagnostic Standards for Metabolic Syndrome. In Japanese. Nippon Naika Gakkai Zasshi, 94, 794-809. doi:10.2169/naika.94.794

[8] Miyatake, N., Nonaka, K. and Fujii M. (1999) A new air displacement plethysmograph for the determination of Japanese body composition. Diabetes, Obesity and $\mathrm{Me}$ tabolism, 1, 347-351. doi:10.1046/j.1463-1326.1999.00064.X

[9] Mccrory, M.A., Gomez, T.D., Bernauer, E.M. and Mole, P.A. (1995) Evaluation of a new air displacement plethysmograph for measuring human body composition. Medicine \& Science in Sports \& Exercise, 27, 1686-1691. doi:10.1249/00005768-199512000-00016

[10] Miyatake, N., Wada, J., Nishikawa, H., Saito, T., Takenami, S., Miyachi, M., Makino, H. and Numata, T. (2007) Comparison of muscle strength between Japanese men with and without metabolic syndrome. Acta Med Okayama, 66, 99-102.

[11] Kigawa, A., Yamamoto, T., Koyama, Y., Kageyama, S. and Arima, K. (1987) Evaluation of knee extensor strength for prevention of sports injury. In Japanese, Japanese Orthopaedic Society of Sports Medicine, 6, 141-145.

[12] Jurimae, T., Hurbo, T. and Jurimae, J. (2009) Relationship of handgrip strength with anthropometric and body composition variables in prepubertal children. Homo, 60, 225238. doi:10.1016/j.jchb.2008.05.004

[13] Koley, S. and Singh, A.P. (2009) An association of dominant hand grip strength with some anthropometric variables in Indian collegiate population. Anthropologischer Anzeiger, 67, 21-28. doi:10.1127/0003-5548/2009/0003

[14] Luna-Heredia, E., Martin-Pena, G. and Ruiz-Galiana, J. (2005) Handgrip dynamometer in healthy adults. Clinical Nutrition, 24, 250-258. doi:10.1016/j.clnu.2004.10.007

[15] Miyatake, N., Miyachi, M., Tabata, I. and Numata, T. (2009) Leg strength per body weight is associated with ventilatory threshold in Japanese women. Anti-Aging Medicine, 6, 5-9.

[16] Miyatake, N., Takenami, S., Kawasaki, Y. and Fujii, M. (2004) Relationship between visceral fat accumulation and physical fitness in Japanese women. Diabetes Research and Clinical Practice, 64, 173-179. doi:10.1016/j.diabres.2003.11.004 